\title{
Configuration-Sensitive Molecular Sensing on Doped Graphene Sheets
}

\author{
John Russell and Petr Král ( $₫)$ \\ Department of Chemistry, University of Illinois at Chicago, Chicago, IL 60607, USA \\ Received: 11 February 2010 / Revised: 6 April 2010 / Accepted: 29 April 2010 \\ C The Author(s) 2010. This article is published with open access at Springerlink.com
}

\begin{abstract}
We show by molecular dynamics simulations that configuration-sensitive molecular spectroscopy can be realized on optimally doped graphene sheets vibrated by an oscillatory electric field. High selectivity of the spectroscopy is achieved by maximizing Coulombic binding between the detected molecule and a specific nest, formed for this molecule on the graphene sheet by substituting selected carbon atoms with boron and nitrogen dopants. One can detect binding of different isomers to the nest from the frequency shifts of selected vibrational modes of the combined system. As an illustrative example, we simulate detection of hexanitrostilbene enantiomers in chiral nests formed on graphene.
\end{abstract}

\section{KEYWORDS}

Molecular sensing, enantiomer, graphene, functionalization, molecular dynamics

\section{Introduction}

Selective molecular sensing is important in numerous areas, such as military [1], fire sensing [2], wine tasting [3], and cancer detection [4]. Recent advances in nanotechnology have brought new possibilities to develop accurate, reliable, and portable chemical sensors, which may benefit a broad range of industries [5, 6]. Among numerous tested systems, carbon nanostructures $[7,8]$ and, in particular, carbon nanotubes (CNT) allow the preparation of highly sensitive sensors [9-16]. The molecules adsorbed on the CNT surfaces are sensed via changes of the CNT quasi-one-dimensional conductivity [17], but the approach is usually less specific, due to the nonspecific character of molecular binding to CNTs.

Molecular sensing can also be realized with vibrating microstructures which measure the mass of adsorbed

$\overline{\text { Address correspondence to pkral@ }}$ uic.edu molecules [18]. CNTs have been tested as vibrational sensors of attached molecules [19, 20], where the molecules can be sensed with Raman scattering $[21,22]$. Suspended and vibrated graphene monolayers may also be used for this purpose [23, 24]. Although $\mathrm{CNT}$ and graphene-based sensors might be highly sensitive, they cannot easily distinguish between isomers.

In this work, we describe highly selective graphenebased vibrational sensors that can detect molecules in different isomeric states. Our idea is to form selective nests for polar molecules on small graphene sheets by selectively replacing carbon atoms with boron and nitrogen dopants [25]. The dopants are positioned to create an electrostatic field above the graphene that is complementary to that of the detected (attached) molecule, so that their Coulombic binding is maximized. Once attached in the nest, the molecule can be 
distiguished from its isomers by the frequency shifts of the normal vibrational modes of the combined graphene-molecule system.

Several approaches might be potentially used to experimentally realize such nests. For example, scanning tunneling microscopy (STM) has been shown to induce local reactions [26]. STM might be used to reactively replace selected $\mathrm{C}$ atoms with $\mathrm{B}$ and $\mathrm{N}$ atoms in the graphene. Alternatively, STM might form the nests by attaching charged (polar) atomic [27-29] or molecular ligands $[30,31]$ to different regions of the graphene [32,33]. One could also externally synthesize molecules that are complementary to the tested molecules, attach them covalently to the graphene, and vibrate the combined system [34].

\section{Design of the molecular nest}

We demonstrate these ideas for a hexanitrostilbene (HNS) sensor. First, we calculate the electronic structure and optimized geometry of HNS using Gaussian03 [35] at the B3LYP/STO-3G level. The atomic charges are calculated using the natural bond order (NBO) approach. As shown in Fig. 1(a), HNS is composed of two trinitrobenzyl groups connected via a trans double bond. In the ground state, HNS is chiral since these two groups are twisted with respect to one another, like the blades of a molecular propeller [36].

In Fig. 1(b)-1(c) the calculated electrostatic potential of HNS is presented in the $(x, y)$ plane with a fixed distance $(z=-2.35 \AA$ and $3.15 \AA$ ) below (Fig. 1(b)) and above (Fig. 1(c)) above (bottom left) and below (bottom right) the geometric center of the molecule, which is located at $z \approx-0.166 \AA$ below the central C-C double bond. Slight (ground-state) bending of the HNS in the central region and related reorganization of the $-\mathrm{NO}_{2}$ groups result in the HNS having $C_{2}$ symmetry along the $z$ axis but not along the $x$ or $y$ axis. Therefore, the two charged profiles shown in Fig. 1(b)-1(c) are not mirror images of each other. The six highly polar $-\mathrm{NO}_{2}$ groups provide useful sites for recognition. The distinct arrangements of charges in the chiral HNS might in principle be used to distinguish between its two enantiomers [37], with mirror imaged charges.

We design a selective molecular nest on doped graphene for the chosen (M)-HNS enantiomer. We position the molecule above the center of the graphene sheet $(\approx 39.5 \AA \times 24 \AA)$ at a typical binding distance of $z=3.35 \AA$. Then, we start to form the nest under the (M)-HNS by modifying the graphene sheet, considering that each of its $C$ atoms is a potential candidate for replacement by boron or nitrogen. Two $\mathrm{B}$ or two $\mathrm{N}$ atoms are not allowed to be neighbors, and the edge of graphene is left open and without doping. The

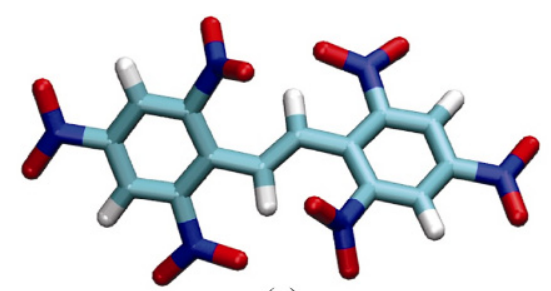

(a)

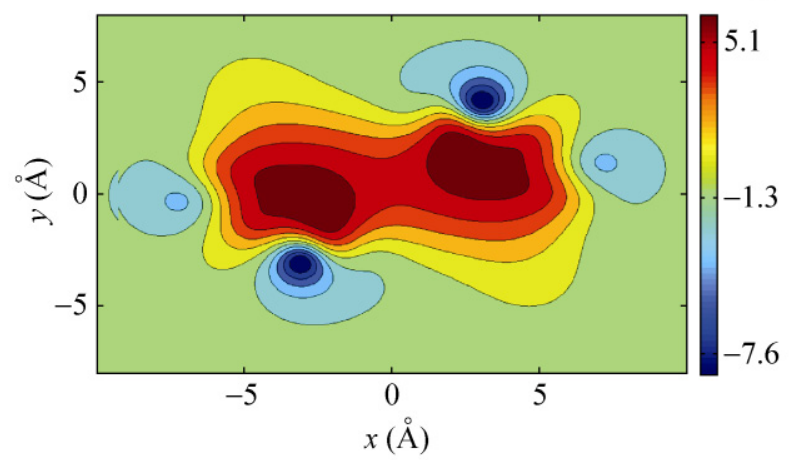

(b)

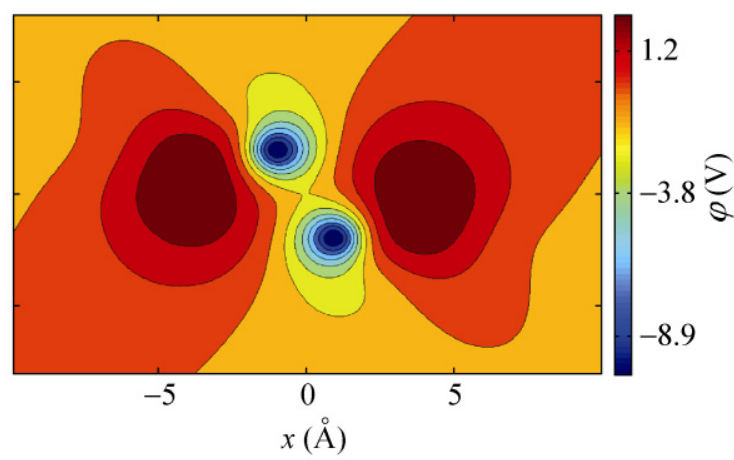

(c)

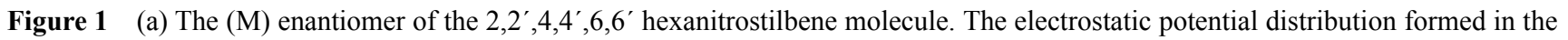
plane located $(b) \approx-2.35 \AA$ below and $(\mathrm{c}) \approx 3.15 \AA$ above the geometric center of the HNS molecule 
doping $\mathrm{B}$ and $\mathrm{N}$ atoms in the sheet have the charge of $Q_{\mathrm{B}} \approx 0.55 \mathrm{e}$ and $Q_{\mathrm{N}} \approx-0.55 \mathrm{e}$, which is approximately (exactly in the model) neutralized by the charges on the three neighboring $\mathrm{C}$ atoms. The energies associated with the substitutions of $\mathrm{C}$ with $\mathrm{B}$ and $\mathrm{N}$ atoms are given by the formula $\Delta E_{\mathrm{B}(\mathrm{N})}=E_{\text {doped }}+E_{\mathrm{C}}-E_{\text {undoped }}-E_{\mathrm{B}(\mathrm{N})}$. Both reactions are endothermic and (in a $78 \mathrm{C}$-atom flake) require $\Delta E_{\mathrm{B}}=4.58 \mathrm{eV}$ and $\Delta E_{\mathrm{N}}=2.53 \mathrm{eV}$. The NBO charges and substitution energies are obtained using the B3LYP density-functional and $6-31 G^{*}$ basis set within Gaussian03 [35].

When we test for $\mathrm{B}$ or $\mathrm{N}$ doping of a particular site, we calculate the change of potential energy due to Coulombic coupling between the molecule and the neutral cluster formed by the charged dopant and its three oppositely charged neighbors [25]. This change of potential energy is

$$
E_{\mathrm{B}(\mathrm{N})}=Q_{\mathrm{B}(\mathrm{N})} \sum_{i=1}^{n} \frac{Q_{i}}{4 \pi \varepsilon_{0}}\left(\frac{1}{r_{i 0}}-\sum_{j=1}^{3} \frac{1}{3 r_{i j}}\right),
$$

where $r_{i 0}$ is the distance between the $i$ th atom in the molecule, with the charge $Q_{i}$, and the dopant with the charge $Q_{\mathrm{B}(\mathrm{N})}, r_{i j}$ is the distance between this $i$ th atom and the $j$ th neighbor of the dopant, with the charge $\approx-Q_{\mathrm{B}(\mathrm{N})} / 3$, and $\varepsilon_{0}$ is the dielectric constant. We decide to dope a particular site when the change of the Coulombic potential energy decreases at least by the value of $\Delta E=-0.02 \mathrm{eV}$.

In Fig. 2(a), we show the nest formed by doping the graphene sheet under the (M)-HNS. The doping pattern complements the electrostatic fields under the molecule (Fig. 1(c)) and copies its $C_{2}$ symmetry; the exact $C_{2}$ symmetry is achieved by doping the sheet by atom pairs with central symmetry. Figure 2(b) shows the profile of the electrostatic field generated in a $(x, y)$ plane positioned at a distance of $1 \AA$ above (or below) the sheet. The partial positive charges of the boron dopants in the graphene match the partial negative charges of the oxygens in nitro-groups in the (M)-HNS, and the partial negative charges of the nitrogen dopants in the graphene match the partial positive charges of the electron deficient benzene rings in the (M)-HNS. The resulting nest possesses a two-dimensional chirality [38].

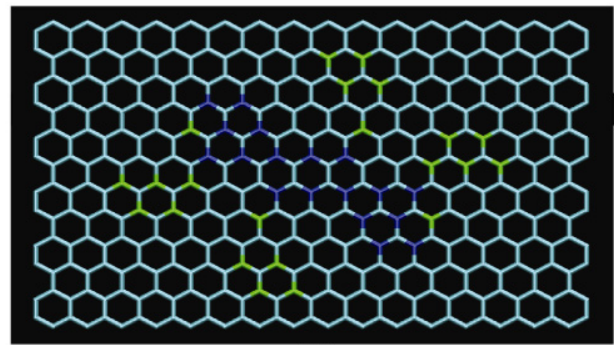

(a)

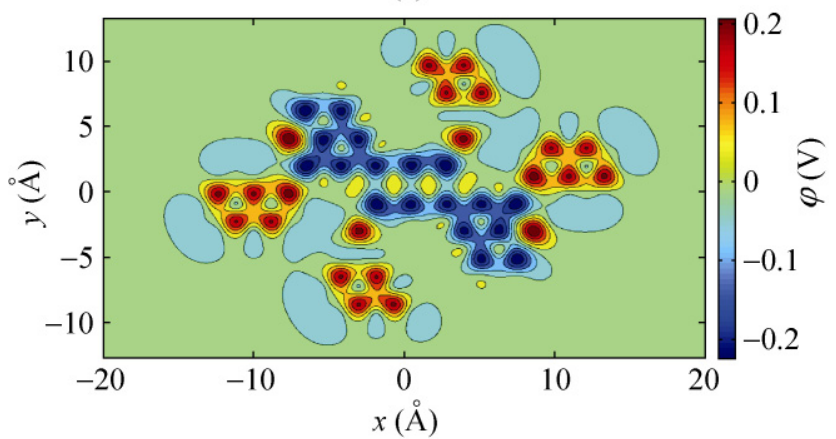

(b)

Figure 2 The doping pattern of the nest designed for the (M)-HNS enantiomer docked above the sheet (a). The electrostatic potential distribution (b) formed above (or below) the doped graphene sheet (complementary to that in Fig. 1(c))

\section{Simulation of selective molecular docking}

Next, we use molecular dynamics (MD) simulations to model nesting and sensing of the HNS on the doped graphene sheet. The simulations are realized with the NAMD package [39], based on the CHARMM27 force field [40]. We use the NVT ensemble without periodic boundary conditions, where Langevin damping is used to thermalize the system and model its vibrational damping, as explained later, and the simulation time step is $1 \mathrm{fs}$. The vibrational properties are described by Lennard-Jones potentials, parametrized in HNS as in the trinitrobenzyl molecule [41], while graphene is parametrized by CHARMM27, where $k_{\text {bond }}=305 \mathrm{kcal} / \AA^{2}, K_{\text {angle }}=40 \mathrm{kcal} /\left(\mathrm{mol} \cdot \mathrm{rad}^{2}\right)$, and $K_{\text {dihedral }}=3.1 \mathrm{kcal} / \mathrm{mol}$.

In Fig. 3, we show that the (M)-HNS enantiomer can properly dock $(T=75 \mathrm{~K})$ in the chiral nest only from the top side of the graphene sheet. On the top, the (M)-HNS lays in the nest, while on the bottom, it stands like a "scorpio". Therefore, by blocking the bottom side, the nest matches only the (M)-HNS enantiomer. This enantioselectivity is caused by the 


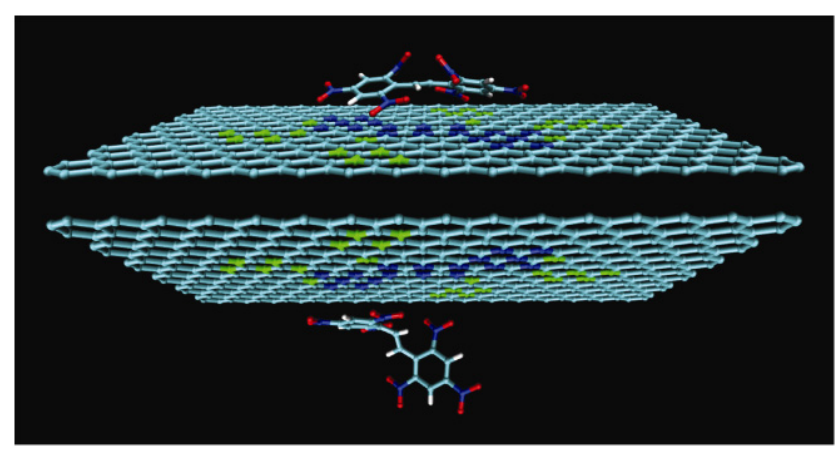

Figure 3 The (M)-HNS enantiomer docked in the nest on the graphene sheet designed for it (top). However (bottom), the (M)-HNS enantiomer does not bind well to the nest at the bottom of the graphene sheet, because this nest (with its vertically mirrored electric field) matches the opposite (P)-HNS enantiomer

fact that the bottom of the sheet creates an electric field that is a mirror image (in the $z$ axis) of that above the sheet. Therefore, this field matches a mirror imaged molecule, i.e., the (P)-HNS enantiomer. The (M)-HNS enantiomer can still bind to it, but its nesting configuration is very different, which might be recognized by vibrational means.

We calculate the binding energy of the nested (M)-HNS, shown in Fig. 3 (top). Initially, we center the molecule in the nest and allow the system to relax for $t=25 \mathrm{ps}$ at the temperature of $T=75 \mathrm{~K}$. At this temperature the (M)-HNS has enough energy to rapidly explore the doped graphene, but once docked it shows only very small vibrational motion. By using VMD [42], we obtain (from 225 ps long simulations) the total (electrostatic, van der Waals (vdW), and HNS configuration) binding energy of the (M)-HNS to the top of the nest of $\Delta E_{\text {nest- } \mathrm{t}} \approx-39.1 \mathrm{kcal} / \mathrm{mol}$, while to the bottom of the nest it is $\Delta E_{\text {nest-b }} \approx-30.2 \mathrm{kcal} / \mathrm{mol}$, and its binding energy to the undoped graphene is $\Delta E_{\text {graphene }} \approx-28.2 \mathrm{kcal} / \mathrm{mol}$. Due to the large difference of $\Delta E=\Delta E_{\text {nest-t }}-\Delta E_{\text {graphene }} \approx-10.9 \mathrm{kcal} / \mathrm{mol}$, the (M)-HNS remains (Coulombically) bound to the top of the nest at $T=300 \mathrm{~K}$.

For practical reasons, it is interesting to find out if the (M)-HNS nests on its own. When it is displaced from the nest by $20 \AA$ in the $x$-axis and $5 \AA$ in the $z$-axis, it diffuses back to the nest after $\tau_{\text {nest }} \approx 200$ ps $(T=75 \mathrm{~K})$ (see the movie in the Electronic Supplementary Material). We also test if other residual molecules can stay in the nest at room temperature. It turns out that water molecules leave it at $T=300 \mathrm{~K}$. Similarly, we tested binding to the nest of stilbene, an achiral molecule similar to HNS but with hydrogen atoms replacing the nitro groups. The binding energy is $\Delta E_{\text {nest }} \approx-27.3 \mathrm{kcal} / \mathrm{mol}$, while its binding energy to the undoped graphene is $\Delta E_{\text {graphene }} \approx-18.0 \mathrm{kcal} / \mathrm{mol}$. Therefore, the (M)-HNS binds by $\Delta E \approx-1.6 \mathrm{kcal} / \mathrm{mol}$ more strongly to the nest than stilbene, despite the similarity of the two structures. These results show that when various molecules are captured in the nest, in practice only the (M)-HNS can remain there at higher temperatures.

\section{Simulation of molecular sensing}

We start the MD simulations of molecular sensing by comparing the elastic properties of our model graphene with experiments. First, we calculate the Young's modulus of graphene defined as $E=\sigma / \varepsilon$, where $\sigma$ is the stress and $\varepsilon$ the applied strain [43]. We clamp one side of the graphene sheet and exert an in-plane stretching force on the edge opposite the clamped side. The induced stress is given by $\sigma=n F / w_{\mathrm{g}} t_{\mathrm{g}}$, where $n$ is the number of atoms with force $F$ acting on each of them, $t_{\mathrm{g}} \approx 3.4 \AA$ is the thickness (interlayer separation of graphite) and $w_{\mathrm{g}}$ is the width of the graphene $(y \approx 24 \AA)$. Likewise, the strain is $\varepsilon=\Delta l / l_{0}$, where $\Delta l$ is the change in length of the graphene, with the original length $l_{0}$, due to the force acting on it. We find that our model graphene has the Young's modulus of $E \approx 1 \mathrm{TPa}$, in good agreement with the experimental value of $E \approx 1 \mathrm{Tpa} \pm 0.1 \mathrm{TPa}$ [44].

Next, we determine the flexural rigidity $D$ of our model graphene by rolling it into a cylinder and calculating the difference in configuration energies [45]. The associated energy density, $W=\frac{1}{2} D \kappa^{2}$, can be used to obtain the flexural rigidity $D$ from the curvature of the rolled graphene, $\kappa=R^{-1}$. Our graphene gives $D \approx 4.5\left(\mathrm{eV} \cdot \AA^{2}\right) /$ atom, in a reasonable agreement with ab initio results, giving $D \approx 3.9\left(\mathrm{eV} \cdot \AA^{2}\right) /$ atom $[46,47]$. Therefore, the elastic parameters of our model graphene should match the real values.

We continue with modeling the (M)-HNS sensing in a nest formed on a small piece of doped graphene, shown in Fig. 2 (a), and clamped at the shorter sides. 
In experiments, the sheet can be suspended over a gap, clamped on two edges, and vibrated by an oscillatory electric field applied to an electrode located below the sheet $[48,49]$. The highly polarizable graphene sheet becomes periodically attracted to the electrode and mechanically oscillated. The vibration amplitude can reflect the presence of the molecule in the nest, since the effective mass and thus the frequency of selected normal vibrational modes are shifted.

We model sensing of the (M)-HNS nested on a doped graphene sheet that is driven by an oscillatory force of $F(t)=A \sin (\omega t) \cdot \theta(t)$, where $\theta(t)$ is the Heaviside step function. For simplicity, the force is applied in the $z$ direction to each of the six carbon atoms in the center of the graphene sheet. The vibration of the graphene sheet may be approximately characterized by the absolute displacement of each atom in the $z$ direction (neglecting the $x$ and $y$ displacements). We can model the excitation of normal modes in the clamped graphene sheet as driven and damped harmonic oscillators. The displacement in the $z$ direction in the center of the sheet (or other selected points) can be described for each normal mode by the equation,

$$
m_{\mathrm{e}} \ddot{z}(t)=b \dot{z}(t)+k z(t)=F(t),
$$

where $m_{\mathrm{e}}$ is the effective mass of the oscillator (normal mode), $b$ is its damping constant and $k$ is its force constant. Eq. (2) has the solution [50],

$$
\begin{aligned}
z(t)= & \theta(t) A \mathrm{e}^{-\delta t} \frac{2 \delta \omega \omega_{\mathrm{d}} \cos \left(\omega_{\mathrm{d}} t\right)+\omega \Delta_{-} \sin \left(\omega_{\mathrm{d}} t\right)}{\left(\omega_{0}^{2}-\omega^{2}\right)^{2}+4 \delta^{2} \omega^{2}} \\
& +\theta(t) A \frac{-2 \delta \omega \omega_{\mathrm{d}} \cos (\omega t)+\omega_{\mathrm{d}} \Delta_{+} \sin \left(\omega_{\mathrm{d}} t\right)}{\left(\omega_{0}^{2}-\omega^{2}\right)^{2}+4 \delta^{2} \omega^{2}}
\end{aligned}
$$

where $\Delta_{ \pm}=\delta^{2}+\omega^{2} \pm \omega_{\mathrm{d}}^{2}, w_{0}=\sqrt{k / m_{\mathrm{e}}}$ is the intrinsic frequency, $\delta=b / 2 m_{\mathrm{e}}$ is the effective damping, and $\omega_{\mathrm{d}}=\sqrt{\omega_{\mathrm{d}}^{2}-\delta^{2}}$ is the resonant frequency of the damped oscillator.

The coefficient $\delta$ in Eq. (3) represents the total damping of the chosen normal mode $r$ in the graphene sheet suspended over a gap and clamped at the edges [51]. This damping is caused by coupling of the normal mode to other internal and external (at the contacts) degrees of freedom (vibrational, electronic, etc.) [52]. In our simulations, we approximate this total damping by applying to the system a Langevin damping of
$0.2 \mathrm{ps}^{-1}$, which roughly corresponds to theoretical values obtained for graphene mechanically oscillated at the frequency of $100 \mathrm{GHz}$ [51].

The steady-state component in Eq. (3) (second term), valid for one normal mode, has a resonant character, where the position and width of the resonance could be used to detect the mass of the nested molecule. Its nesting configuration [53] might be also detected, because different normal modes have their effective masses, oscillation maxima, and nodes distributed differently on the graphene. These modes may sense the configurations of the molecule, depending on its nesting position at the maxima and/or nodes of the modes.

The sensitivity of a given mode to the attached molecule can be obtained from the change of its frequency when the molecule is bound. Assuming, for simplicity, that the HNS molecule sits in the "center of the mode vibration", where its mass, $m_{\mathrm{HNS}}$, may simply add to the effective mass of the mode, $m_{\mathrm{e}}$, we obtain for the ratio of the intrinsic frequencies of the two (free and with molecule) oscillators,

$$
\frac{\omega_{\text {unbound }}}{\omega_{\text {bound }}}=\sqrt{1+\frac{m_{\mathrm{HNS}}}{m_{\mathrm{e}}}} .
$$

Here, we assume that the force constant of the mode, $k$, is the same for both the bound and unbound cases. This shows that modes with smaller effective masses $m_{\mathrm{e}}$ are more sensitive, because the HNS mass is a larger perturbation for them. By exciting the system at different driving frequencies, $\omega$, we can detect the modified resonances of the individual modes. Since the driving may simultaneously excite many different modes of finite energy widths, we need to separate their contributions.

\section{Results and discussion}

We illustrate these ideas by detecting the (M)-HNS nested on both sides of the doped graphene. We use the "axial" and "edge" normal vibrational modes of the clamped graphene sheet, shown in the insets of Figs. 4 and 5, respectively. First, we obtain the "reference frames" of these two modes by exciting the empty sheet, held on its two edges, at two frequencies that 


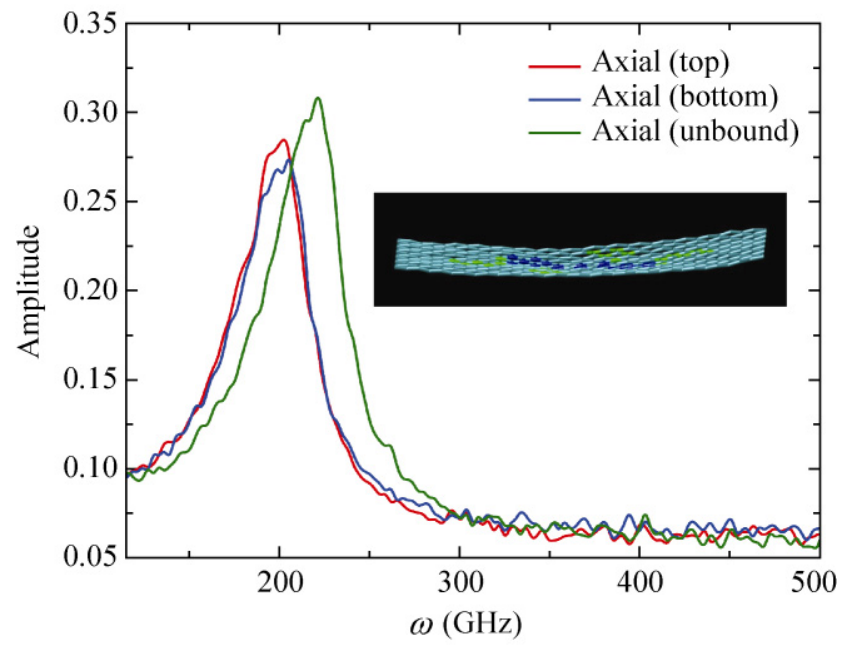

Figure 4 The frequency dependent values of the projected amplitude (arbitrary units) of the axial vibration mode $(T=75 \mathrm{~K})$ We display the vibrations of the unbound sheet (green), and the sheet with the (M)-HNS bound at the top (red) and bottom (blue). The inset shows the atom displacements in the reference frame of the axial vibration mode in the clamped graphene sheet $(T=5 \mathrm{~K})$

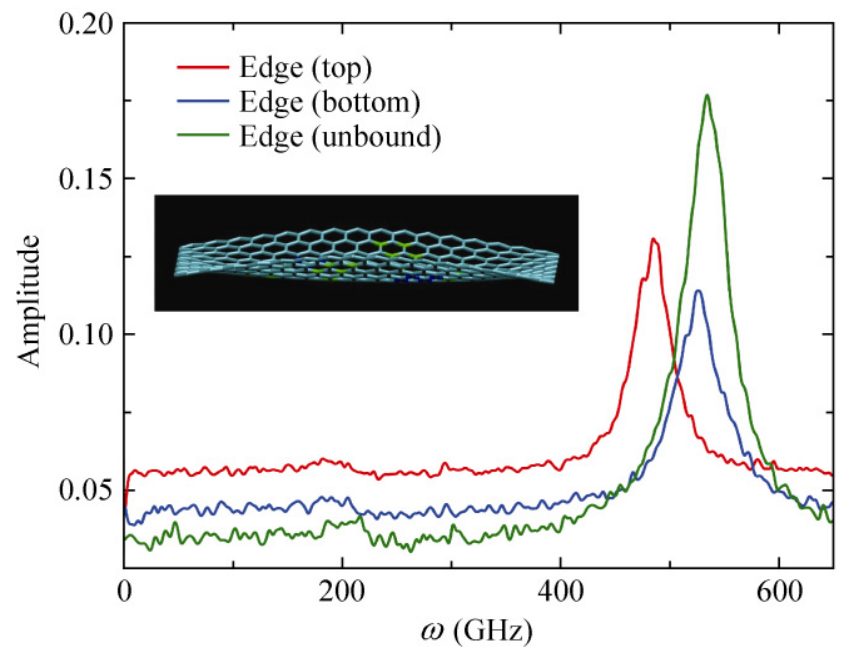

Figure 5 The frequency dependent values of the projected amplitude (arbitrary units) of the edge vibration mode $(T=75 \mathrm{~K})$. We display the vibrations of the unbound sheet (green), and the sheet with the (M)-HNS bound at the top (red) and bottom (blue). The inset shows the atom displacements in the reference frame of the edge vibration mode in the clamped graphene sheet $(T=5 \mathrm{~K})$

are close to their resonances. In the initial simulations, realized at $T=5 \mathrm{~K}$, we pick frames with a maximum atom displacement and use them as the reference frames, shown in Figs. 4 and 5. The frames are obtained after relaxing the transient component in the solution (Eq. (3)). The axial mode corresponds to the displacement of the graphene sheet, as in transverse acoustic (TA) phonon modes with waves propagating along the sheet [54]. The edge mode has the same type of displacement but the TA waves propagate in the orthogonal direction, where the graphene edges are free.

In the simulations of the molecular detection (realized at $T=75 \mathrm{~K}$ ), we relax the transient component in Eq. (3) and find the oscillatory contributions of these two modes to the total vibration of the graphene sheet. We approximately separate the amplitude of vibrations of the modal type $r$ at frequency $\omega, A_{\mathrm{r}}(\omega)$, by projecting the actual frames on the reference frame of the chosen mode,

$$
A_{\mathrm{r}}(\omega)=\frac{1}{N_{\mathrm{f}}} \sum_{i=1}^{N_{\mathrm{f}}}\left|\sum_{j=1}^{N_{\mathrm{a}}} z_{j}^{\mathrm{si}}(\omega) z_{j}^{\mathrm{r}}\left(\omega_{\mathrm{r}}\right)\right| .
$$

Here, $N_{\mathrm{f}}$ is the number of frames sampled from the molecular dynamics trajectory and used in the averaging, and $N_{\mathrm{a}}$ is the number of atoms in the sheet. $z_{j}^{\text {si }}(\omega)$ are the $z$-coordinates of atom $j$ in the simulation frame $i$ at frequency $\omega$. Likewise, $z_{j}^{\mathrm{r}}(\omega)$ are the $z$-coordinates of atom $j$ in the reference frame of mode $r$ at the modal resonant frequency $\omega_{\mathrm{r}}$. From the $A_{\mathrm{r}}(\omega)$ amplitude we can obtain the position and width of the resonance of the mode $r$.

In our simulations, the force $F$ of the amplitude $A=$ $10.4 \mathrm{pN}$ is applied on each of the six $\mathrm{C}$ atoms, and its frequency ranges in the interval of $\omega=1-900 \mathrm{GHz}$, in stepwise increments of $3 \mathrm{GHz}$. Each frequency step is held constant for $0.5 \mathrm{~ns}$ comprised of 2000 frames. The first 100 frames $(0.025 \mathrm{~ns})$ are discarded in order to eliminate transient vibrations caused by the change in frequency (the first term in Eq. (3)). The remaining 1900 frames ( $0.475 \mathrm{~ns})$ per frequency step are analyzed, as shown in Eq. (5).

In Fig. 4, we show the results of our simulations of (M)-HNS sensing realized by the axial mode at $T=75 \mathrm{~K}$; this low temperature is chosen to reduce the noise level of the vibrating system. We compare frequency dependent values of the projected amplitude obtained for the vibrations of the free sheet (unbound case), and the sheet with the (M)-HNS bound at the top and bottom. The full width at half-maximum (FWHM) of $52-55 \mathrm{GHz}$ is similar in all three cases and fixed mostly by the Langevin damping. On the other hand, the 
resonant frequencies are shifted from the unbound value of $\omega_{\text {unbound }} \approx 220 \mathrm{GHz}$ to the values of $\omega_{\text {bound }} \approx$ $202 \mathrm{GHz}$ and $\omega_{\text {bound }} \approx 205 \mathrm{GHz}$ for the case when the (M)-HNS is bound above and below the graphene, respectively. Although, the last two frequencies are similar, we can distinguish between the two cases. From the unbound and bound (above graphene) frequencies, and the HNS mass, $m_{\mathrm{HNS}} \approx 450 \mathrm{~g} / \mathrm{mol}$, we can obtain, using Eq. (4), the effective mass of the axial mode, $m_{\mathrm{e}} \approx 2419 \mathrm{~g} / \mathrm{mol}$.

Let's now evaluate the same spectra for the edge mode, as shown in Fig. 5. In this case, the resonance shifts from the unbound-case frequency of $\omega_{\text {unbound }} \approx$ $535 \mathrm{GHz}$ (FWHM $\approx 48.2 \mathrm{GHz}$ ) to $\omega_{\text {bound }} \approx 484 \mathrm{GHz}$ $(\mathrm{FWHM} \approx 46.1 \mathrm{GHz})$ and $\omega_{\text {bound }} \approx 526 \mathrm{GHz}(\mathrm{FWHM} \approx$ $53.1 \mathrm{GHz}$ ) for the case when the HNS is bound above and below the graphene, respectively. We can also find the effective mass of the edge mode, $m_{\mathrm{e}} \approx 2027 \mathrm{~g} / \mathrm{mol}$, using Eq. (4). In this case, we can not only clearly detect the presence of the bound (M)-HNS, but we can also see where it is bound. Therefore, if only one side of the graphene is exposed, we could distinguish between the two HNS enantiomers.

The above results show that the edge mode is much more sensitive than the axial mode in the detection of the HNS configurations. This is because (1) the effective mass, $m_{\mathrm{e}}$ of the edge mode is smaller than that of the axial mode and (2) the fact that the different (M)-HNS configurations influence very differently the resonance in the edge mode. In the axial mode, the configuration of the (M)-HNS molecule does not matter much, because the molecule is in both cases (above and below) close to the maximum of mode oscillations. On the other hand, in the edge mode, when the (M)-HNS is above the graphene, it is close to the maximum of the oscillations, while when it sits below the graphene it is positioned very close to the nodal point of this mode. In principle, we can use this type of spectroscopy to fully recover the average orientation of the nested molecules.

In summary, we have shown that polar molecules may be selectively captured by electrostatic nests formed on doped graphene sheets. Their presence and docking configurations can be detected by the described configuration-sensitive spectroscopy from the shifts of resonant frequencies in selected vibrational modes of the graphene sheet. With this spectroscopy, one can detect and possibly sort the chirality of enantiomers by vibrational means, when attached to flat but chiral surfaces.

\section{Acknowledgements}

JR would like to acknowledge the generous support from the National Defense Science and Engineering Fellowship sponsored by the Department of Defense.

Electronic Supplementary Material: A movie showing that when the (M)-HNS is displaced from the nest it diffuses back to the nest after $\tau_{\text {nest }} \approx 200$ ps (at $T=75 \mathrm{~K}$ ) is available in the online version of this article at http://dx.doi.org/10.1007/s12274-010-0007-7 and is accessible free of charge.

Open Access: This article is distributed under the terms of the Creative Commons Attribution Noncommercial License which permits any noncommercial use, distribution, and reproduction in any medium, provided the original author(s) and source are credited.

\section{References}

[1] Kunz, R. R.; Gregory, K. C.; Hardy, D.; Oyler, J.; Ostazeski, S. A; Fountain, A, W. III. Measurement of trace explosive residues in a surrogate operational environment: implications for tactical use of chemical sensing in C-IED operations. Anal. Bioanal. Chem. 2009, 395, 357-369.

[2] Ni, M.; Stetter, J. R.; Buttner, W. J. Orthogonal gas sensor arrays with intelligent algorithms for early warning of electrical fires. Sensor. Actuat. B: Chem. 2008, 130, 889-899.

[3] Li, R.; Wang, P.; Hu, W. L. A novel method for wine analysis based on sensor fusion technique. Sensor. Actuat. B: Chem. 2000, 66, 246-250.

[4] Willis, C.; Church, S.; Guest, C.; Cook, W.; McCarthy, N.; Bransbury, A.; Church, M.; Church, J. Olfactory detection of human bladder cancer by dogs: Proof of principle study. Brit. Med. J. 2004, 329, 712-715.

[5] Modi, A.; Koratkar, N.; Lass, E.; Wei, B. Q.; Ajayan, P. M. Miniaturized gas ionization sensors using carbon nanotubes. Nature 2003, 424, 171-174.

[6] Zhong, Z. H.; Wang, D. L.; Cui, Y.; Bockrath, M. W.; Lieber, C. M. Nanowire crossbar arrays as address decoders for integrated nanosystems. Science 2003, 302, 1377-1379. 
[7] Kong, J.; Franklin, N. R.; Zhou, C. W.; Chapline, M. G.; Peng, S.; Cho, K.; Dai, H. J. Nanotube molecular wires as chemical sensors. Science 2000, 287, 622-624.

[8] Hu, J. T.; Odom, T. W.; Lieber, C. M. Chemistry and physics in one dimension: Synthesis and properties of nanowires and nanotubes. Acc. Chem. Res. 1999, 32, 435-445.

[9] Freitag, M.; Johnson, A. T.; Kalinin, S. V.; Bonnell, D. A. Role of single defects in electronic transport through carbon nanotube field-effect transistors. Phys. Rev. Lett. 2002, 89, 216801.

[10] Staii, C.; Johnson, A. T. Chen, M.; Gelperin, A. DNAdecorated carbon nanotubes for chemical sensing. Nano Lett. 2005, 5, 1774-1778.

[11] Chen, R. J.; Bangsaruntip, S.; Drouvalakis, K. A; Kam, N. W. S.; Shim, M.; Li, Y. M.; Kim, W.; Utz, P. J.; Dai, H. J. Noncovalent functionalization of carbon nanotubes for highly specific electronic biosensors. P. Natl. Acad. Sci USA 2003, 100, 4984-4989.

[12] Snow, E. S.; Perkins, F. K.; Houser, E. J.; Reinecke, T. L. Chemical detection with a single-walled carbon nanotube capacitor. Science 2005, 307, 1942-1945.

[13] Qi, P. F.; Vermesh, O.; Grecu, M.; Javey, A.; Wang, Q.; Dai, H. J.; Peng, S.; Cho, K. J. Toward large arrays of multiplex functionalized carbon nanotube sensors for highly sensitive and selective molecular detection. Nano Lett. 2003, 3, 347-351.

[14] Williams, K. A.; Veenhuizen, P. T. M.; de la Torre, B. G.; Eritja, R.; Dekker, C. Nanotechnology: Carbon nanotubes with DNA recognition. Nature 2002, 420, 761.

[15] Wang, F.; Gu, H. W.; Swager, T. M. Carbon nanotube/ polythiophene chemiresistive sensors for chemical warfare agents. J. Am. Chem. Soc. 2008, 130, 5392-5393.

[16] Zhang, T.; Nix, M. B.; Yoo, B. Y.; Deshusses, M. A.; Myung, N. V. Electrochemically functionalized single-walled carbon nanotube gas sensor. Electroanal. 2006, 18, 1153-1158.

[17] Dan, Y. P.; Lu, Y.; Kybert, N. J.; Luo, Z. T.; Johnson, A. T. Intrinsic response of graphene vapor sensors. Nano Lett. 2009, 9, 1472-1475.

[18] Ilic, B. R.; Krylov, S.; Kondratovich, M.; Craighead, H. G. Optically actuated nanoelectromechanical oscillators. IEEE J. Quantum Electr. 2007, 13, 392-399.

[19] Garcia-Sanchez, D.; San Paulo, A.; Esplandiu, M. J.; PerezMurano, F.; Forro, L.; Aguasca, A.; Bachtold, A. Mechanical detection of carbon nanotube resonator vibrations. Phys. Rev. Lett. 2007, 99, 088501.

[20] Li, C. Y.; Chou, T. -W. Mass detection using carbon nanotube-based nanomechanical resonators. Appl. Phys. Lett. 2004, 84, 5246-5248.

[21] Jorio, A.; Dresselhaus, G.; Dresselhaus, M. S.; Souza, M.;
Dantas, M. S. S.; Pimenta, M. A.; Rao, A. M.;Saito, R.; Liu, C.; Cheng, H. M. Polarized Raman study of single-wall semiconducting carbon nanotubes. Phys. Rev. Lett. 2000, 85, 2617-2620.

[22] Casiraghi, C.; Hartschuh, A.; Qian, H.; Piscanec, S.; Georgi, C.; Fasoli, A.; Novoselov, K. S.; Basko, D. M.; Ferrari, A. C. Raman spectroscopy of graphene edges. Nano Lett. 2009, 9 , 1433-1441.

[23] Schedin, F.; Geim, A. K.; Morozov, S. V.; Hill, E. W.; Blake, P.; Katsnelson, M. I.; Novoselov, K. S. Detection of individual gas molecules adsorbed on graphene. Nat. Mater. 2007, 6, 652-655.

[24] Bunch, J. S.; van der Zande, A. M.; Verbridge, S. S.; Frank, I. W.; Tanenbaum, D. M.; Parpia, J. M.; Craighead, H. G.; McEuen, P. L. Electromechanical resonators from graphene sheets. Science 2007, 315, 490-493.

[25] Wang, B. Y.; Král, P. Optimal atomistic modifications of material surfaces: Design of selective nesting sites for biomolecules. Small 2007, 3, 580-584.

[26] Katano, S.;Kim, Y; Hori, M; Trenary, M; Kawai, M. Reversible control of hydrogenation of a single molecule. Science 2007, 316, 1883-1886.

[27] Balog, R.; Jorgensen, B. J.; Nilsson, L.; Andersen, M.; Rienks, E.; Bianchi, M.; Fanetti, M.; Laegsgaard, E.; Baraldi, A.; Lizzit, S., et al. Bandgap opening in graphene induced by patterned hydrogen adsorption. Nat. Mater. 2010, 9, 315-319.

[28] Li, J. -L.; Kudin, K. N.; McAllister, M. J.; Prudhomme, R. K.; Aksay, I. A.; Car, R. Oxygen-driven unzipping of graphitic materials. Phys. Rev. Lett. 2006, 96, 176101.

[29] Xu, Z. P.; Xue, K. Engineering graphene by oxidation: A first-principles study. Nanotechnology 2010, 21, 045704.

[30] Liu, Z.; Robinson, J. T.; Sun, X. M.; Dai, H. J. PEGylated nanographene oxide for delivery of water-insoluble cancer drugs. J. Am. Chem. Soc. 2008, 130, 10876-10877.

[31] Stankovich, S.; Dikin, D. A.; Dommett, G. H. B.; Kohlhaas, K. M.; Zimney, E. J.; Stach, E. A.; Piner, R. D.; Nguyen, S. T.; Ruoff, R. S. Graphene-based composite materials. Nature 2006, 442, 282-286.

[32] Wang, X. R.; Li, X. L.; Zhang, L.; Yoon, Y.; Weber, P. K.; Wang, H. L.; Guo, J.; Dai, H. J. N-doping of graphene through electrothermal reactions with ammonia. Science 2009, 324, 768-771.

[33] Wang, H. L.; Wang, X. R.; Li, X. L.; Dai, H. J. Chemical selfassembly of graphene sheets. Nano Res. 2009, 2, 336-342.

[34] Li, C.; Qian, X. G.; Yang, B. J.; Yan, Y.; Qian, Y. T. Synthesis and characterization of nitrogen-rich graphitic carbon nitride. Mater. Chem. Phys. 2007, 103, 427-432.

[35] Frisch, M. J.; Trucks, G. W.; Schlegel, H. B.; Scuseria, G. E.; Robb, M. A.; Cheeseman, J. R.; Montgomery, J. A.; 
Vreven, T.; Kudin, K. N.; Burant, J. C. et al. Gaussian 03, Revision C.02, Gaussian, Inc., Wallingford CT (2004).

[36] Wang, B. Y.; Král, P. Chemically tunable nanoscale propellers of liquids. Phys. Rev. Lett. 2007, 98, 266102.

[37] Xu, W.; Jin, L.; Lu, Y. X.; Lan, B. J.; Zou, Z. G. $\mathrm{C}_{3}$-symmetric molecules with axial chirality and handed arrangement of dipole fields. Chem. Res. Chinese U. 2007, 23, 628-930.

[38] Ernst, K. -H. Supramolecular surface chirality. Top. Curr. Chem. 2006, 265, 209-252.

[39] Phillips, J. C.; Braun, R.; Wang, W.; Gumbart, J.; Tajkhorshid, E. ; Villa, E.; Chipot, C.; Skeel, R. D.; Kale, L.; Schulten, K. Scalable molecular dynamics with NAMD. J. Comput. Chem. 2005, 26, 1781-1802.

[40] MacKerell, A. D.; Bashford, D.; Bellott, M.; Dunbrack, R. L.; Evanseck, J. D.; Field, M. J.; Fischer, S.; Gao, J.; Guo, H.; Ha, S., et al. All-atom empirical potential for molecular modeling and dynamics studies of proteins. J. Phys. Chem. $B$ 1998, 102, 3586-3616.

[41] Michael, D.; Benjamin, I. Molecular dynamics simulation of the water-nitrobenzene interface. J. Electroanal. Chem. 1998, 450, 335-345.

[42] Humphrey, W.; Dalke, A.; Schulten, K. VMD: Visual molecular dynamics. J. Mol. Graph. 1996,14, 33-38.

[43] Chou, P. C.; Pagano, N. J. Elasticity Tensor, Dyadic, and Engineering Approaches; Van Nostrand: Princeton, 1967.

[44] Lee, C.; Wei, X. D.; Kysar, J. W.; Home, J. Intrinsic strength of monolayer graphene. Science 2008, 321, 385-388.

[45] Lu, Q.; Arroyo, M.; Huang, R. Elastic bending modulus of monolayer graphene. J. Phys. D: Appl. Phys. 2009, 42, 102002.
[46] Arroyo, M.; Belytschko, T. Finite crystal elasticity of carbon nanotubes based on the exponential Cauchy-Born rule. Phys. Rev. B 2004, 69, 115415.

[47] Kudin, K.; Scuseria, G. E. $\mathrm{C}_{2} \mathrm{~F}, \mathrm{BN}$, and $\mathrm{C}$ nanoshell elasticity from ab initio computations. Phys. Rev. B 2001, 64, 235406.

[48] Chen, C. Y.; Rosenblatt, S.; Bolotin, K. I.; Kalb, W.; Kim, P.; Kymissis, I.; Stormer, H. L.; Heinz, T. F.; Hone, J. Performance of monolayer graphene nanomechanical resonators with electrical readout. Nat. Nanotechnol. 2009, 4, 861-867.

[49] Naik, A. K.; Hanay, M. S.; Hiebert, W. K.; Feng, X. L.; Roukes, M. L. Towards single-molecule nanomechanical mass spectrometry. Nat. Nanotechnol. 2009, 4, 445-450.

[50] Morse, P. M.; Ingard, K. U. Theoretical Acoustics, 1st ed.; Princeton University Press: Princeton, 1986.

[51] Geller, M. R.; Varley, J. B. Friction in nanoelectromechanical systems: Clamping loss in the $\mathrm{GHz}$ regime. 2005, arXiv: cond-mat/0512710. arXiv.org e-Print archive. http://arxiv.org/ abs/cond-mat/0512710v1 (accessed Jan 23, 2010).

[52] Seoanez, C.; Guinea, F.; Neto, A. H. C. Dissipation in graphene and nanotube resonators. Phys. Rev. B 2007, 76, 125427.

[53] Wang, B.; Král, P.; Thanopulos, I. Docking of chiral molecules on twisted and helical nanotubes: Nanomechanical control of catalysis. Nano Lett. 2006, 6, 1918-1921.

[54] Garcia-Sanchez, D.; van der Zande, A.; San Paulo, A.; Lassagne, B.; McEuen, P.; Bachtold, A. Imaging mechanical vibrations in suspended graphene sheets. Nano Lett. 2008, 8 , 1399-1403. 